\title{
Design and Test of Sensor for Aircraft Engine Gas Path Debris Electrostatic Monitoring
}

\author{
Wei Liu ${ }^{1,2, *}$, Manguo Huang ${ }^{1,2}$, Defeng Liu ${ }^{1,2}$, Yanshan Wang ${ }^{1,2}$, Meiju Zhang ${ }^{1,2}$ \\ ${ }^{1}$ Beijing Changcheng Aeronautic Measurement and Control Technology Research Institute, Beijing 101111, China \\ ${ }^{2}$ Aviation Key Laboratory of Science and Technology on Special Condition Monitoring Sensor Technology, Beijing 101111, \\ China
}

\begin{abstract}
The research and design of sensor for aircraft engine gas path debris electrostatic monitoring are given in this paper. The mechanism of charged debris and principle of electrostatic induction of sensor are researched firstly, and when the charged material near or keep away from the electrostatic sensor, the induced charge of opposite polarity will be induced on the sensor's probe. And then, the structure of electrostatic monitoring sensor is designed based on the principle of electrostatic induction. Electrostatic sensor is constituted by sensitive element, dielectric, internal circuit and grounding shell. Due to the particularity of working environment of electrostatic sensor, various factors such as performance requirements and using occasions should be considered in designed process. Finally, the effectiveness of electrostatic monitoring sensor is verified using experimental system. From the experimental results we can know when the charged particles through the electrostatic sensor, the sensing signal amplitude and the activity level are change obviously. These works provide theoretical method for the early fault warning of aircraft engine gas path components, and the monitoring and management of aircraft engine working condition.
\end{abstract}

\section{Introduction}

In 1970s, the U.S. air force institute of technology found that when the aircraft engine gas path components fault occurs, the electrical activity in the gas path channel will increase [1]. When aircraft engine combustor jet exhaust, meanwhile, with a lot of electronics, ions and charged particles including the state information of aircraft engine gas path components generated [2]. Using the electrostatic sensor to detect these charged particles can obtain status information of the engine gas path components, which can provide early warning information for engine. In the 1990s, Powrie and Fisher et al studied the technology and method of using the state of the electrostatic sensors to monitor engine. This method has been used successfully in the $U$. S. joint fighter F-35 [3-4]. The current domestic research on gas path electrostatic sensor monitoring technology just started, a lot of researched results of the engine gas path simulated test [5-6] and engine's gas path electrostatic monitoring signal [7-8] has been achieved, but the current domestic public research mainly based on the ground of a certain type of test-platform of aircraft engine. As the main research target, the engine exhaust gas electrostatic signal was studied, but the research of electrostatic sensor that is used to monitor charged particle is less public. In this paper, the electrostatic sensor for aircraft engine gas path debris detection have been researched, the principle of electrostatic monitoring was analyzed, the model and the design of structure are given, and the effectiveness of the proposed sensor was verified by the simulated research finally.

\section{The Principle of Electrostatic Sensor}

\subsection{The mechanism of charged particles}

Apart from carbon dioxide, nitrogen, water vapor, hydrocarbon, carbon monoxide, sulfur oxides and produced by incomplete combustion of carbon particles and other normal particles, the exhaust of aircraft engine may also include abnormal particles because of the fault of engine's gas path components. There are a lot of reasons for the abnormal particles appeared in the exhaust gas, for example, the suction inlet of an alien, the falling blade caused by local instantaneous high temperature, disbonding, the loss of leaf material caused by cyclical stress under the local environment of high temperature, excessive carbon smoke particles in chamber caused by local rich oil, as well as the rubbing between blade and casing or sealing ring.

In aircraft engine combustion chamber, the high temperature from the burning fuel oil makes the gas molecules to occur the chemical ionization reaction, so there are many charged ions owing to the gassy chemical ionization reaction in the gas path. When the carbon smoke particles formed in the rich oil region pass through the high-temperature flame zone, interaction between the free electrons and ions formed by chemical

\footnotetext{
*Corresponding author: 1w880623@126.com
} 
ionization make the particles charged [9]. The smoke from the burning of particle collide with the random thermal motional ions under the environment of high temperature, which can produce electric charge, the process can be expressed as:

$$
q_{p}=\frac{d_{p} k T}{2 e^{2}} \ln \left(1+\frac{d_{p} c \pi e^{2} N t}{2 k T}\right)
$$

In the equation, $q_{p}$ is the particle charge, $d_{p}$ is the particle diameter, $k$ is the Boltzmann constant, $T$ is temperature, $e$ is the electronic charge, $c$ is the average speed of particles, $N$ is particle concentration, $t$ is time.

\subsection{Principle of electrostatic sensor}

When a charged object closes to or keep far away from a non-charged conductor, there will be induced charge on the surface of conductor and the phenomenon is called electrostatic induction. The basic principle of electrostatic induction is that the charged objects can produce electric field in space, and the charge will redistribute within the electric force of the conductor and then induced current generated inside the sensor. Electrostatic sensor principle diagram is shown in Fig. 1. When the charged material near or keep away from the electrostatic sensor, the induced charge of opposite polarity will be induced on the sensor's probe.
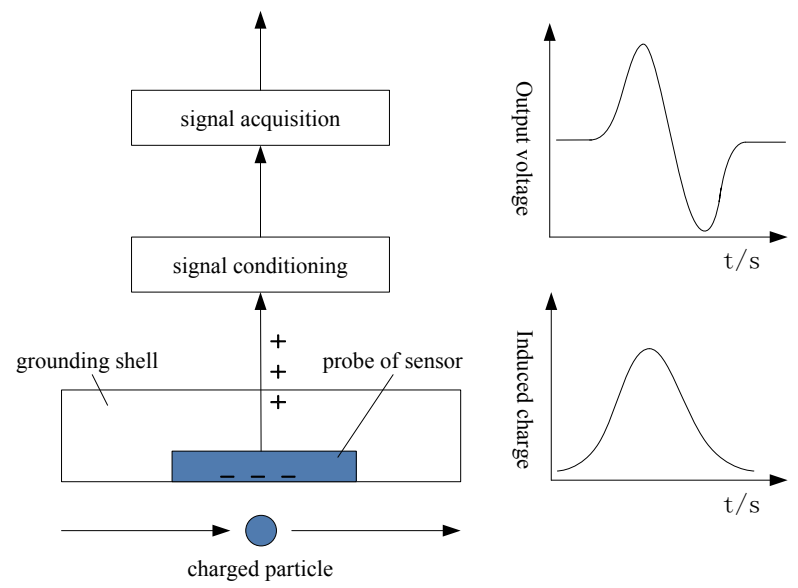

Figure 1. Schematic diagram of induced charge and current.

Assuming that the induced charge on the probe is $q(t)$, the measurement of the output voltage can be represented by equivalent circuit shown in Fig. 2.



Figure 2. Measuring equivalent circuit.
In Fig. 2, $R$ represents the equivalent resistance measuring circuit, $C$ represents the equivalent capacitance measuring circuit. Assuming that the initial state of the circuit are zero, then the relationship between the Laplace transform $U(S)$ of output voltage and the Laplace transform $Q(S)$ of induced charge are:

$$
U(S)=\frac{R S Q(S)}{1+R C S}
$$

When $\mathrm{RCS}<<1$, the measurement of model equals to:

$$
u(t)=R q^{\prime}(t)
$$

The equation reflects the changes of the induced charge.

\section{Design of the Electrostatic Sensor}

\subsection{Constitution of electrostatic sensor}

Electrostatic sensor is constituted by sensitive element, dielectric, internal circuit and grounding shell, as shown in Fig. 3. When charged particles flow through the measuring electrode, it will induce the charge equal in size, symbol instead on the proximal and the distal. The induced amount of charge is all the sum of the induced charge in electrostatic sensor. You can take single charged particles as point charge here. The induced characteristic will emerge when the point charge pass through the measuring electrode, reflecting their own sensing characteristics of the measuring electrode also. The mathematical models of electrostatic sensor are established on the basis of the point charge in this paper.

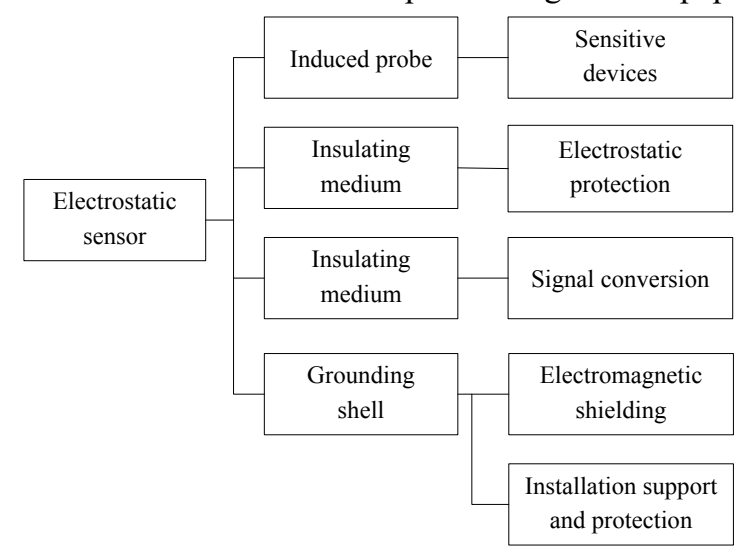

Figure 3. Structure and function model diagram of electrostatic sensor.

\subsection{Design of electrostatic sensor}

Due to the particularity of working environment of electrostatic sensor, various factors such as performance requirements and using occasions should be considered in designed process. The probe is put in the nozzle directly. Not only the materials of probe should satisfy 
the sensitive induction electric field but also the high temperature characteristic. The isolation materials between probe with grounding shell material behave conductive not easily and generate electrostatic charge not easily, and have the characteristics of high temperature resistant as same as the mat.

The design of electrostatic sensor is shown in Fig. 4. In this figure, 1 is the stainless steel plug, 2 is the stainless steel shell, 3 is the ceramic plate, 4 is ceramic sleeve, and 5 is nickel base alloy probe.

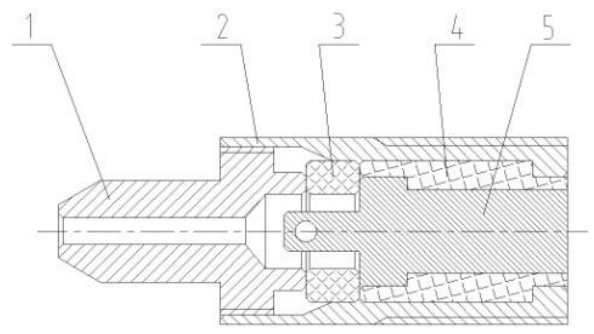

Figure 4. Structure of electrostatic sensor.

The plug and the shell are made by stainless steel with the characteristic of high temperature resistance. The shell mainly has the effect of external electromagnetic interference shielding, protecting the internal circuit structure at the same time, and grounded well. Cover and sleeve are made by ceramic material with good insulated performance and high temperature resistance, the main effect of cover and sleeve is to prevent the interference of external static and the loss of internal static signal. The probe is made by conductive easily nickel base alloy material with the characteristic of high temperature resistance, ensuring the good conductivity. The extended wire of electrostatic sensor is made by single core shielded wire.

\section{Analysis of Experiment}

To verify the effectiveness of the designed electrostatic sensor, the electrostatic monitoring experiment of simulated engine pipeline particles was done, the experimental system is shown in Fig. 5.

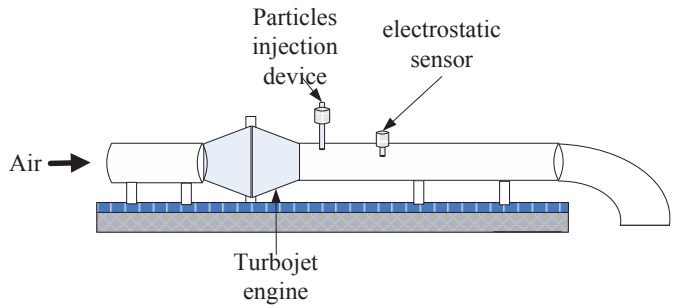

Figure 5. Simulation experiment system of electrostatic sensor.

The whole tested system consists of particles injection device, electrostatic sensor, small turbojet engine system, exhaust pipe, exhaust device, installation of platform. The electrostatic sensor installed on the exhaust pipe. The particles injection device is used to inject simulated particles to exhaust pipe, the small turbojet engine system is used to simulate the hightemperature environment.

In the process of experiment, the turbojet engine was opened firstly, and the iron powder particles were injected into the pipeline periodically. The particles sampled signal using electrostatic sensor is shown in Fig. 6.From this figure we can see that the sampled signal amplitude has an obvious change when the iron powder particles were injected, and the peak to peak value is increased obviously.

The activity level (AL) of sampled signal is shown in Fig. 7. From this figure we can know that the activity level of particles sampled signal is higher than the background signal, which indicates the electrostatic sensor can detect the charged particles effectively.

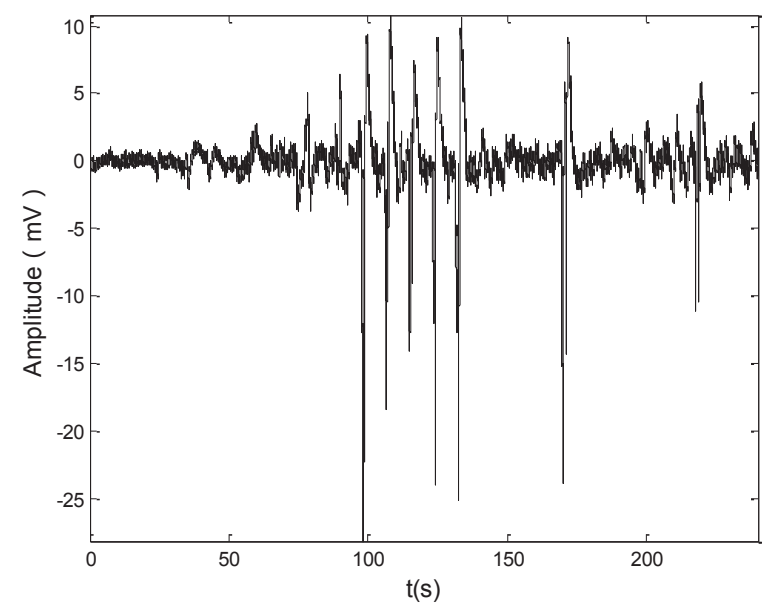

Figure 6. Particles sampled signal.

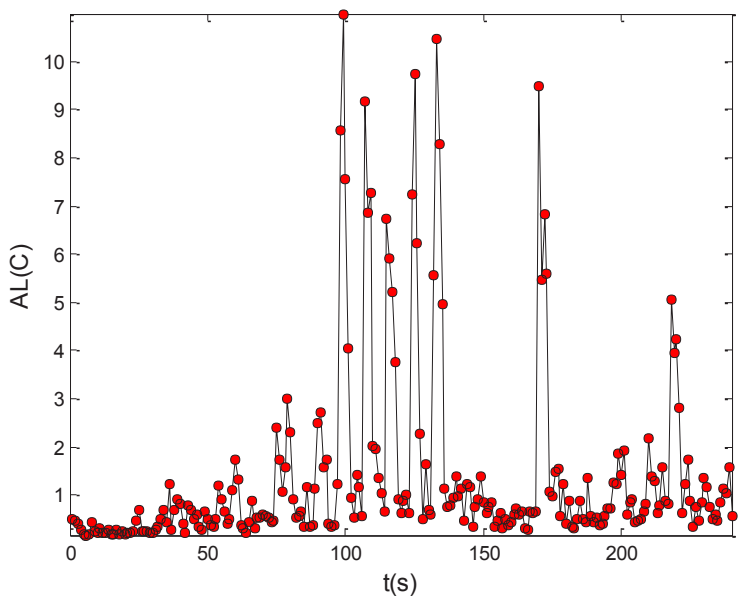

Figure 7. Activity level.

The activity level (AL) is defined as:

$$
A L(t)=\sqrt{\frac{1}{T} \int_{0}^{T} Q_{f}^{2}(t) d t}=\sqrt{\frac{1}{N} \sum_{n=1}^{N} Q_{f_{n}}^{2}}
$$

In the equation, $Q_{f}$ is the electrostatic charge signal, $T$ is the intercepted signal length, usually takes for $1 \mathrm{~s}, N$ 
is the sampling points in $T$ after discrete sampling for $Q_{f}$, and $Q_{f_{n}}$ is the electrostatic charge signal after discrete sampling for $Q_{f}$.

\section{Conclusions}

Based on the principle of electrostatic induction sensor, the design of electrostatic sensor is given, and the electrostatic monitoring experiment was processed. From these analyses, we can obtain the following conclusion: When the charged particles through the electrostatic sensor, the sensing signal amplitude and the activity level are change obviously, which verifies the effectiveness of the electrostatic sensor designed in this paper.

\section{Acknowledgment}

The author thanks all those who helped her during the process of writing this manuscript and during her research. This work was supported by the Aeronautical Science Fund 2014ZD34005.

\section{References}

1. H. E. G. Powrie, K.Mcnicholas, Gas path condition monitoring during accelerated mission testing of a demonstrator engine, AIAA paper, 972904 (1997)

2. A. Sorokin, F. Arnold, Electrically charged small soot particles in the exhaust of an aircraft gas turbine engine combustor: comparison of the model and experiment, Journal of Atmospheric Environment, 38, 17 (2004)

3. H. E. G. Powrie, A Novis. Gas path debris monitoring for $\mathrm{F}-35$ to be strike fighter propulsion system PHM, Aerospace Conference Proceedings, 2006 Montana, USA, 11 (2006)

4. C. E. Fisher, Gas path debris monitoring - a 21 st century PHM tool, Aerospace Conference Proceedings, 2006 Montana, USA, 6 (2006)

5. Li Yaohua, Zuo Hongfu, Wen Zhenhua, the Simulated Experiment of Aircraft Engine Gas Path Debris Monitoring Technology, ACTA AERONAUTICA ET AS TRONAUTICA SINICA, , 30,4 (2009)

6. Li Yaohua, Zuo Hongfu, Method for Rub Fault Detection Based on Electrostat ic Technique : Model and Simulated Experiments, ACTA AERONAUTICA ET ASTRONAUTICA SINICA, 31, 6 (2010)

7. Liu Pengpeng, Zuo Hongfu, Fu Yu, Sun Jianzhong. Exhaust gas electrostatic monitoring and gas path fault feature for turbojet engine, Journal of Aerospace Power, 28, 2 (2013)

8. Sun Jianzhong, Zuo Hongfu, Liu Pengpeng, Yu Youfeng, and Chen Zhixiong, "Analysis and application of baseline model of aero-engine exhaust gas electrostatic monitoring signals," Journal of Aerospace Power, 28, 3 (2013)

9. Ren Jun, Shen Jian, Lu Shouci. The science and technology of particle dispersion , Beijing: chemical industry press, (2005) 